IOSR Journal of Engineering (IOSRJEN)

ISSN: 2250-3021 Volume 2, Issue 6 (June 2012), PP 16-22

www.iosrjen.org

\title{
The Design of Intelligent Campus Security \& Attendance System Based on RFID, GSM and Zig-Bee
}

\author{
${ }^{1}$ V.B.Gopala Krishna ${ }^{2}$ S. Chandra Sekhar ${ }^{3}$ N. Rajesh Babu \\ ${ }^{4}$ K.Sreenivasa Ravi \\ ${ }^{I}$ Dept. of ECE, Sree Vahini Institute of Science \& Tech., Tiruvuru, Krishna Dist, Andhra Pradesh. \\ ${ }^{2}$ H.O.D of ECE Dept, Sree Kavitha College of Engg, Karepally, Khammam Dist, Andhra Pradesh. \\ ${ }^{3}$ Research scholar in KL university Dept of ECE, A.P, India, \\ ${ }^{4}$ Professor in KL university Dept of ECE, A.P, India,
}

\begin{abstract}
ABSRACT: In this paper an intelligent campus security tracking system (iCST) has been designed and implemented base on the RFID and ZigBee network. iCST reads the RFID tags data through FRID\&ZigBee node, and then sends it to PC node by a custom wireless protocol on the ZigBee. PC node gives corresponding warning or hints by the result of matching masterslave RFID tag information. When the warning occurs, its user can logon the web system to get the real-time tracking for valuables (with embedded slave RFID); where the thief arrives any one access control node, it would be blocked. User can also manage his own valuables such as lending and recovery operation through the Web manager center. In this system we are using GSM(Global System For Mobile).These GSM is used sending a SMS as for absentees students with parents mobile no's. The data base is already stored in the PC. Student is regularly absent with in four day or six days free voice call to call the parents mobile no's by using GSM technology. Accompanied by deployment of large-scale sensor networks, iCST has a good application prospect from the aspect of experimental effect.
\end{abstract}

Index Terms: - ZigBee; Internet of things; Wisdom campus;Tracking management system

\section{I.INTRODUCTION}

As the number of valuables owned by college students increases, means of criminal theft becomes more intelligent and hidden. But students' security awareness is relatively weak, which results in frequent campus thefts and has bad effect on teachers, students and the society[1-2]. To solve the problem, we design an intelligent campus security tracking system based on RFID and ZigBee, which has full range of protection on campus that has small flow of people and wiring is not convenient.

RFID(Radio Frequency Identification), which identifies targets within the target area and obtains relevant data automatically through RF signals, has become welcomed by people gradually since it has been proposed[3-5]. The recognition process can resist harsh environments without human intervention. Based on the technology characteristics and strength of RFID, we combine RFID and campus security tracking system, and use SCM control technology and wireless network technology, to develop an intelligent anti-theft systemwith networking capability. That's to say, combine RFID and ZigBee to build a wireless network which doesn't need rewiring. And its special high efficiency and convenience will reduce the cost of campus security and make campus safer, which will reduce the fluency of campus theft effectively and benefit students and teachers.

Based on RFID and ZigBee, the intelligent campus security tracking system uses physical methods and electronic technology, automatically detects theft in monitoring areas, generates alarm signals, and tracks targets through the detection point of RFID. Anti-theft alarm system is

important facility to prevent robbery, theft, accidents, etc. In the event of emergency, it can show the accurate scene in security control center through sound and light alarm signals to enable emergency measures to be taken. Anti-theft alarm system constitutes a security system together with import and export control system, closed circuit television monitoring system, visitor intercom systems and electronic patrol system [6-8].

\section{II.SYSTEM PRINCIPLE AND ERROR ANALYSIS}

The intelligent campus security tracking system is based on wireless communication services between nodes provided by RFID sensors and ZigBee, and identifies the RFID tags within the region to prevent thefts and track valuables, so as to protect the property of the teachers and students. One of the most important conclusions from the early tests of the new GSM technology was that the new standard should employ Time Division Multiple Access (TDMA) technology. 


\section{A. RFID\&ZigBeen nodes implementation}

As the popularity of RFID sensor technology increases, RFID tags are cheap, and can be reused. Data transmission is convenient and easy, we can deal with tags in accordance with customized encryption algorithm based on user requests[9]. Then combine RFID sensor with ZigBee(referred to as RFID\&ZigBee later), and complete the sensor node data collection work. Transceiver inducts RFID tags, sends the information of the read tags to the micro-processor (FreescaleMC9S12XS128) according to RS232 transmission protocol [10]. The micro-processor sends the data to wireless module to complete data transmission after simply data processing, as shown in Figure.1.

Where ZigBee module provides two-way data transmission services and returns the corresponding control information after passing center node and PC processing. Control information is sent to micro-processor through ZigBee communication module. And the system can give the corresponding early warning tips if the ZigBee module has early warning capability (such as entrance guard).

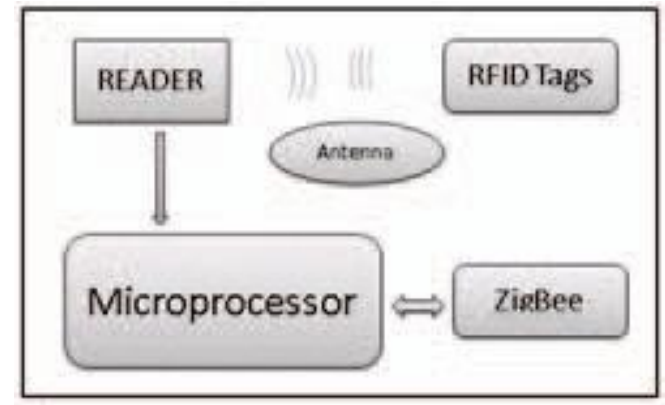

Figure .1 Sensor node schematic of FID \& Zig-Bee

\section{B. Wireless transmission}

The system uses Zig-Bee to build transmission network,

which is used for the transmission of sensor data, and uses customized wireless transmission protocol, which is designed based on simplicity and reliability. In the protocol, considering simplifying microprocessor functions of RFID \& Zig-Bee node and reducing system cost, the wireless transmission protocol mainly achieves the capabilities of error checking, data framing, conflict mechanisms such as retransmission, etc[11].As for error control, considering that tag data is usually short, the

Microprocessor of FRID \& Zig-Bee nodes have strong logic computing power, so the original CRC operation is relatively complex [12] and not suitable for the micro-processor. Therefore, this system innovatively uses new type of customized check method: uses and, or, xor operation for error control, along with length check, and insert frame boundary FLAG into the data frame when sending it.

Assuming the data to be sent is $\mathbf{S} \mathbf{\sim} \boldsymbol{S}$, and source

address(one char), destination address(one char), sequence number(one char), date length(one char), checksum(four char) will be sent with data, as shown in Figure.2(ACK is the same as described above, data section is null, $\mathrm{L}=0$ ).

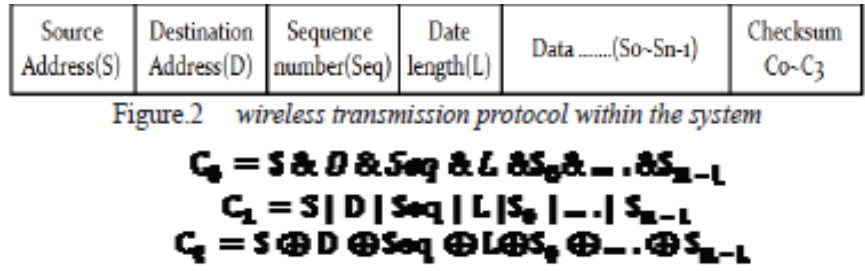

Where the checksum section provides 4 check methods, besides and, or, xor operation, it allows use of stomized method, where 00 means null. The sequence number is mainly used to prevent the additional overhead of retransmission. In the transmitter, the following steps are taken when sending data.

1. Get the data from the upper layer packed, add source address, destination address, sequence number, date length, checksum to it, then send it to the serial output module of the microprocessor. 
The Design of Intelligent Campus Security \& Attendance System Based on RFID, GSM and Zig-Bee

2. The serial output module adds FLAG to the data packet, if FLAG has been in the data, add ESC in front of it. After transmission, start the timer. Go to step 3.

3. Return true if receive ACK. If timeout happens, and timeout time is less than the maximum time, go to step 2; else, return false and inform the upper layer that transmission fails.

When the receiver receives data, perform the following

steps:

1. Check the data packet according to the check operation said above, if check results are the same as those in the data packet, go to step 2; else, drop the packet.

2. See the destination node number of the data packet, if it'sthe same with its ID, go to step 3; else, drop the packet.

3. Return the corresponding ACK of the data packet, determine whether it's request for new packets or retransmitted data packets according to the sequence number of data packet, then send it to the upper layer for computing and handling.

\section{PC node centre and multi-service platform}

The tag information obtained by RFID \& Zig-Bee node is transmitted through Zig-Bee network, and sent to PC node at last. In this system, PC node gets the data of the Zig-Bee network through serial ports, parses out the tag information through customized Zig-Bee protocol. PC node gives the matched results by querying the registered tag information in the database, and returns the corresponding results through the wireless module. When the incoming tag information doesn't match, PC node will give warning message, pass the warning and control message to entrance guard and inform the corresponding owner.

In the multi-service platform, users can view the location of their valuables through multi-service platform interface. When valuables are lost, users can find the course of corresponding valuables through web, so as for tracking and getting it back.

Multi-service platform only gives prompts warning case in this system, but in real life it can achieve anti-theft and monitoring work through functions reservation and system interface.

Specific functions design is shown in Figure. 3

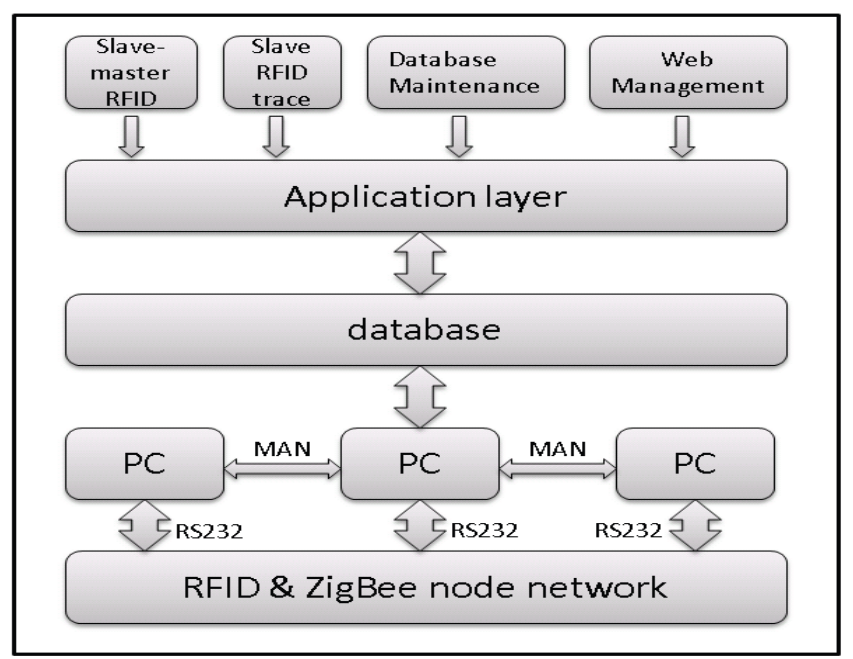

Figure.3 structure of PC node centre service

As can be seen from Figure.3, each PC node is connected through Metropolitan area networks(MAN), thus improving the compatibility of the system greatly. When the RFID sensor network is more widespread, it will greatly improve the tracking range of items.

\section{A. Layout of sensor network}

\section{III.SYSTEM DESIGN AND IMPLEMENTATION}

Implement RFID \& Zig-Bee sensor network layout within the campus, finish transmission through the Zig-Bee protocol, set those important nodes(such as the campus house door, the dormitory door)as PC nodes, which are database maintenance, WEB maintenance, RFID information processing servers. And all PC nodes are connected by Metropolitan area networks (MAN). 
The Design of Intelligent Campus Security \& Attendance System Based on RFID, GSM and Zig-Bee After applying for services, user can get a RFID (Master) tag as a sign of the owner and multiple RFID (slave) tags as signs of valuables. Insert RFID (slave) tag in valuables, and this system can achieve the capability of anti-theft and track lost valuables within the scope of monitoring.

Below is the campus sensor network layout in simulation environment. Set the PC-nodes in the dormitory, classroom building (there exists entrance guard at the location of PC nodes, when master RFID and slave RFID don't match, a warning will be sent.).In real life, RFID \& Zig-Bee nodes should be set on the campus as evenly as possible.

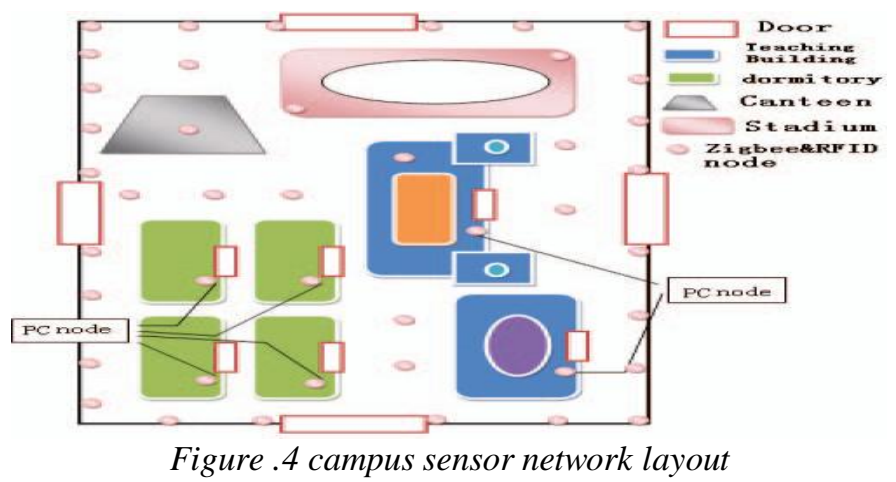

As shown in Figure .4, there are ZigBee \& RFID nodes and PC nodes on the campus, achieving the "Internet of Things" on the campus. PC nodes achieve the collection and processing of server information and database maintenance and query. There is alarm system in front of every door, once it finds that master RFID and slave RFID don't match, it'll give an alarm immediately and inform guards. As for the web form of information display platform, it's also expected to use the campus thumbnails, and words describe the specific location of searched valuables simultaneously.

\section{B. System Architecture and flow chart}

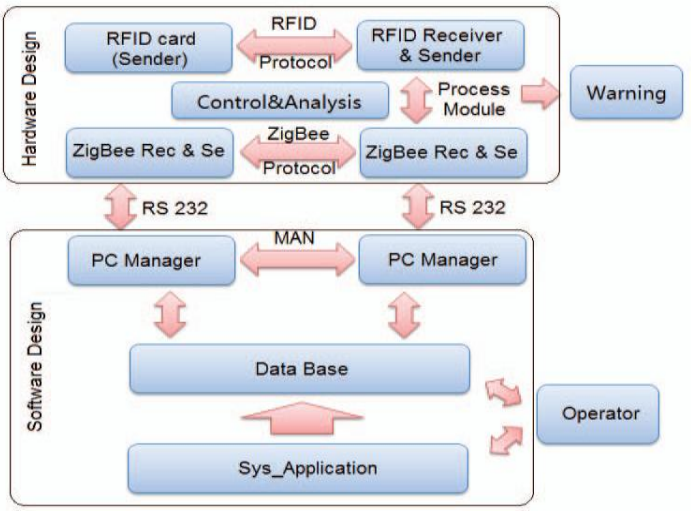

Figure.5 System Architecture chart

As shown in figure.5, the whole system includes two levels design: hardware and software. Hardware includes RFID readers and ZigBee wireless transmission module, and actually micro-processors of FRID \& ZigBee nodes are responsible for connecting those two. On the one hand, read the RFID tag data within the RFID reader sensor area, and then send it to PC node in accordance with the wireless transmission protocol. On the other hand, micro-processors in the location of access control should receive message from the computer and give the prompt warning when mismatched RFID tag passes the system.

Overall, the workflow of the system can be described by the following steps.

1. RFID \& ZigBee nodes sense the RFID (master / slave) labels, send information in the labels to the ZigBee network real time, which is then transferred to the PC nodes. PC checks the label information in the database, when master label and slave label match or only master label appears, the system recognizes it as legal input. But when only slave label appears or master label and slave label don't match, the system recognize it as illegal input, then go to step 2 . 
The Design of Intelligent Campus Security \& Attendance System Based on RFID, GSM and Zig-Bee

2. Record the position changes of RFID (slave) the tags, track the slave tags, and show warning at PC nodes. Then query the database, look for the owner of items and send confirming information to the owner through the system.

3. The owner logins WEB to search for items. He can see the real-time location of his valuables according to the hints. After confirmation, the valuables will be stopped at the entrance guard. As for false information, the owner can cancel this warning.

\section{GSM Technology}

One of the most important conclusions from the early tests of the new GSM technology was that the new standard should employ Time Division Multiple Access (TDMA) technology. The digital nature of GSM allows the transmission of data (both synchronous and asynchronous) to or from ISDN terminals, although the most basic service support by GSM is telephony. A unique feature of GSM is the Short Message Service (SMS), which has achieved wide popularity as what some have called the unexpected 'killer application' of GSM. SMS is a bi-directional service for sending short alphanumeric message in a store-and-forward process. SMS can be used both 'point-to-point' as well as in cell-broadcast mode. These GSM is used sending a SMS as for absentees students with parents mobile no's. The data base is already stored in the PC. Student is regularly absent with in four day or six days free voice call to call the parents mobile no's by using GSM technology.

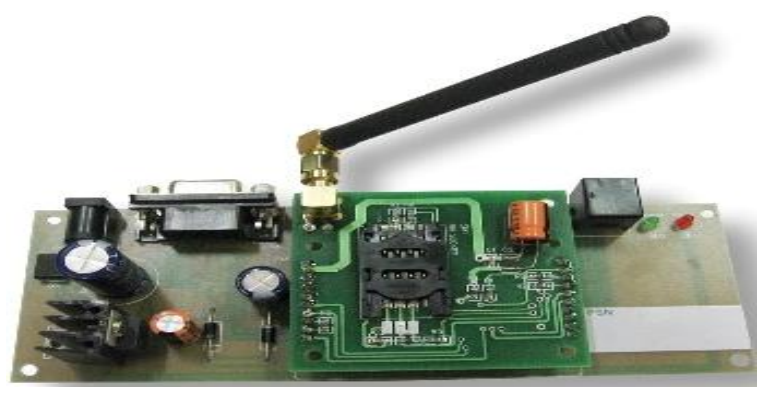

IV. TEST RESULT ANALYSIS

In practice, our system's sensor network uses Middle distance erfid reader, SZ05-STD wireless ZigBee, standard RS232, and the mc9s12xs128 of FreeScale as microprocessor of FRID \& ZigBee node, .and uses Pentium(4) in the XP operation system to do a small-scale tracking and system verification. In order to save experiment cost, we use simulation method to achieve large-scale test and verification of our sensor network. The simulation in the experiment only replaces the process of reading the RFID tag information, and PC directly simulates the actual tag data acquisition process.

As shown in Figure .6, the system detects an illegal input, and produces early warning information. After receiving a reminder, user logs on the system and sees the following warning tips. From the figure, we can see that it's supported to

lend his items to others to enable the daily use of items, so that this warning is cancelled. He can have his items back through this system, too. 


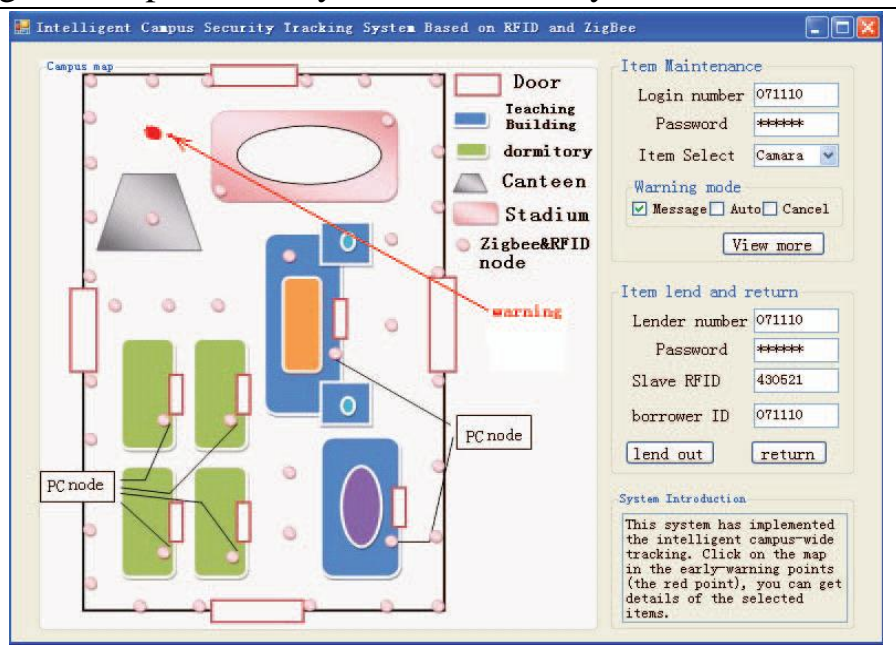

Figure.6 Valuables Management

Users click on the red dot Figure .6, enter the chart of tracking items. Users can choose to confirm or cancel this warning in the tracking figure.

It's worth noting that, if the user does not confirm this early warning, system will check the identity of the suspect at the entrance guard to ensure campus safety, as is shown in Figure.7.

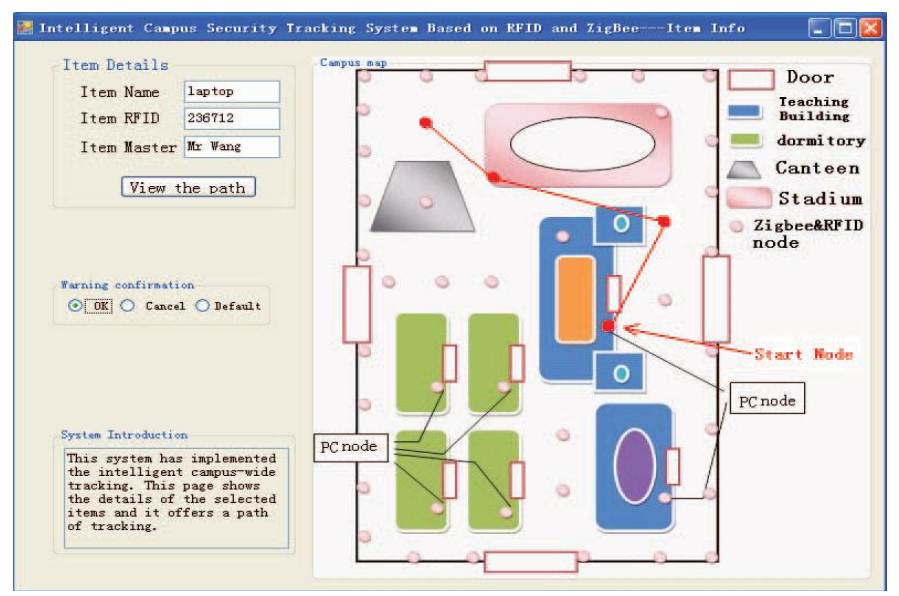

Figure.7 The course of valuables

When the user chooses to confirm or default, valuables will be stopped and checked by the security at the entrance guard so as to achieve security and tracking purposes.

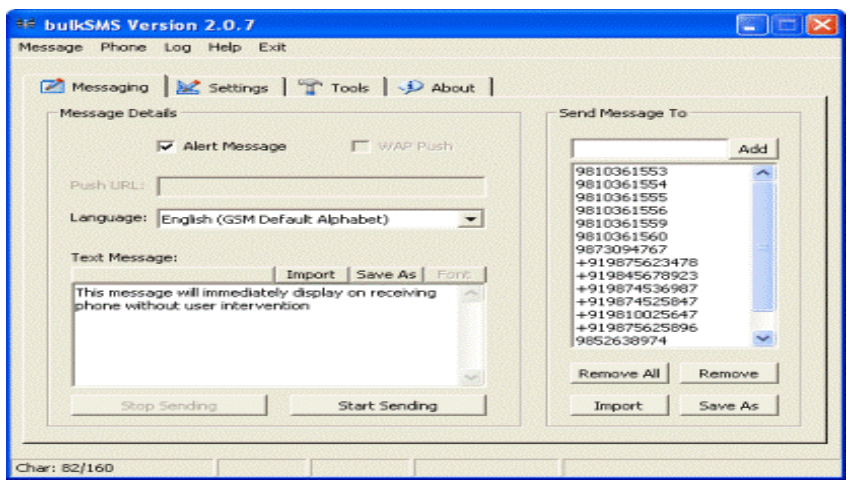

Figure.8 The sending SMS by using GSM technology 


\section{CONCLUSION}

This system studies campus safety and security. By identifying the tags information of master and slave RFID, it not only achieves tracking valuables real time and giving early warning, but also supports users to view the state of their valuables, and lend their valuables to others. As people's awareness of property safety improves, the system can also provide personnel tracking feature to support services related to geographical location. With the further popularization of sensor networks, the system can achieve large-scale logistics management and tracking, and has good market prospects and value in use.

\section{ACKNOWLEDGMENT}

We thank "Intelligent Campus Security Tracking System Based on RFID and ZigBee" project for providing fund support and thank the electronic circuits innovation base of Beijing University of Posts and Telecommunications for providing venues support.

\section{REFERENCES}

[1] Zhu Yuan-jiao, Zhou Ke-qin, Design and Realizing of the Digital Campus Security System, Software Engineering, 2009. WCSE '09. WRI World Congress on IEEE 2009.

[2] Xi Li, Tiyan Shen, Jinjie Zhang, Changmin Shi, A Spatial Technology Approach to Campus Security, Networking, Sensing and Control, 2008. ICNSC 2008. IEEE International Conference on IEEE 2008.

[3] Qian C,Ngan H,Liu Y. Cardinality Estimation for Large-scale RFID Systems[C] .Preceedings of IEEE Int'1 Conf.on Perv.Comp.and Comm(PerCom). 2008, :30-39.

[4] REN Xiao-Kui, LIANG Chao-Zhong, Analysis and Improvement of Anti-Collision Algorithm for RFID System, Computer Systems \& Applications, 2010(2) (in Chinese).

[5] Floerkemeier C. , Sarma S. , An Overview of RFID System Interfaces and Reader Protocols, RFID, 2008 IEEE International Conference.

[6] Zhang Ye, Based on RFID technology jewelry and tracking management system Review, E-Business Journal,2010(9) (in Chinese)

[7] Kuan J.H. , Chang J. , Ho J. , A development of information protection system using system engineering and RFID technolog, System Science and Engineering (ICSSE), 2010 International Conference on IEEE 2010.

[8] ZhuYuYu, WangZengSheng, personnel orientation study Based on the RFID, Science \& Technology Information, 2010(22) (in Chinese).

[9] Chung-Hsin Liu, Jian-Yun Lo, The Study for the ZigBee with RFID Positioning System, Multimedia and Information Technology (MMIT), 2010 Second International Conference on 2010.

[10] Yu Li-e, Deng Xu-dong, The Research about the Application of RFID and 3G Technology in Cargo Transportation Security, Logistics Sci-Tech, 2007(10) (in Chinese).

[11] Elshayeb, S.A., Bin Hasnan, K., Chua Yik Yen, RFID technology and ZigBee networking in improving supply chain traceability Instrumentation, Communications, Information Technology, and Biomedical Engineering (ICICI-BME), 2009 IEEE International Conference. 